\title{
EDITORIAL
}

\section{SPORTS DENTISTRY - CONTRIBUTING TO PERFORMANCE AND HEALTH OF ATHLETES}

Sports Dentistry, although not yet recognized as a specialty by the National Board of Dentistry, has been daily growing and developing in Brazil.

Most people, when asked about this issue, instantly associate it with fabrication of mouthguards or oral trauma. One cannot deny sports practice might increase the risk of oral trauma, especially the contact sports, and dentists play a key role for prevention and resolution of those problems. However, Sports Dentistry presents field of activity much broader and important. Researches show many athletes have poor oral health, with involvement of dental caries, periodontal problems, and dental erosion. Those conditions lead to reduced quality of life and induce systemic inflammatory response, which possibly affects athletes' performance during sports practice.

Dentists also have also an important role in athletes' education concerning prevention and treatment of oral traumas related to sports practice. Studies have shown that many athletes do not wear mouthguards, and they also do not are aware of the protocol procedures in case of trauma, e.g., in scenarios of dental care for tooth avulsion or fractures. Awareness campaigns on the use of mouthguards and oral trauma first aids are of great importance. Similarly, efforts on trauma prevention and education about oral health in athletes are paramount.

Some countries, including the United States, Japan and Brazil, created Sports Dental Associations. Those associations present a multidisciplinary character and act as forum for dentists, physicians, trainers, coaches, nutritionists, speech therapists, and physical therapists that are interested in exchanging ideas and knowledge related to dental needs and risks of injuries of athletes. Several sports clubs already have dentists in their health care personnel, working with the medical team in order to monitor athletes.

In a recent international conference held in São Paulo / Brazil, the importance of dentists in the sports field was extensively presented, with discussions from the prevention aspect to the influence of oral health on performance of highperformance athletes. Thus, a potential working field for dentists is increasingly consolidating in the Dental field, as well as research.

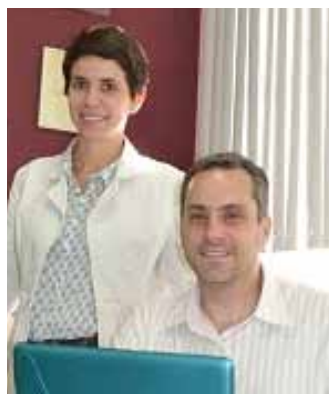

Co-Editors

Eduardo Bresciani

Taciana Marco Ferraz Caneppele 


\section{EDITORIAL}

\section{ODONTOLOGIA ESPORTIVA - CONTRIBUINDO PARAODESEMPENHO E SAÚDE DOS ATLETAS}

A Odontologia Esportiva, embora ainda não reconhecida como Especialidade pelo Conselho Federal de Odontologia, vem crescendo e se desenvolvendo a cada dia no Brasil.

A maioria das pessoas, quando indagadas sobre esse assunto logo associam à confecção de protetores ou traumas bucais. Não se pode negar que a prática esportiva pode aumentar o risco de traumas bucais, em especial os esportes de contato. E, o cirurgião-dentista tem papel fundamental na prevenção e resolução destes problemas. No entanto, a Odontologia Esportiva tem um campo de ação muito mais amplo e importante. Algumas pesquisas mostram que muitos atletas têm uma pobre saúde bucal, com acometimento de cáries, problemas periodontais e erosão dentária. Estas condições podem reduzir a qualidade de vida e induzir a resposta inflamatória sistêmica, podendo afetar seu desempenho durante a prática esportiva.

Em relação a atuação na prevenção e tratamento dos traumas bucais decorrentes da prática esportiva, o cirurgião-dentista tem um papel importante também na educação dos atletas. Estudos mostram que muitos atletas não utilizam protetores bucais, e também não sabem como proceder em caso de um trauma, como por exemplo, os cuidados no caso de avulsão dental ou fraturas. Campanhas de conscientização do uso dos protetores e também de primeiros socorros após traumas bucais são de grande importância. Da mesma maneira, atuar na prevenção e educação em saúde bucal dos atletas é primordial.
Alguns países formaram Associações de Odontologia Esportiva, como Estados Unidos, Japão e Brasil. Estas associações tem um caráter multidisciplinar e funcionam como um fórum de dentistas, médicos, treinadores, técnicos, nutricionistas, fonoaudiólogos, fisioterapeutas interessados em trocar ideias e conhecimentos relacionados às necessidades dentais dos atletas e riscos de injúrias. Vários clubes, já tem um cirurgião-dentista em sua equipe de saúde, trabalhando junto da equipe médica para o acompanhamento dos atletas.

Em recente congresso internacional realizado em São Paulo / Brazil, discutiu-se muito o campo de atuação dos odontólogos na prática esportiva, desde a prevenção até a importância da saúde bucal no rendimento de atletas de alta performance. Assim, uma potencial área de atuação do cirurgião-dentista está a cada dia tomando mais força no mercado, bem como no campo das pesquisas.

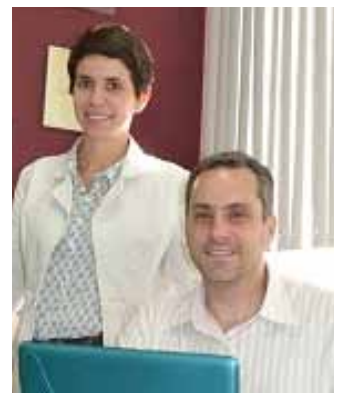

Co-Editors

Eduardo Bresciani

Taciana Marco Ferraz Caneppele 